\title{
The Künneth Formula in Periodic Cyclic Homology
}

\author{
IOANNIS EMMANOUIL \\ Department of Mathematics, The University of Michigan, Ann Arbor, MI 48109-1003, U.S.A.
}

(Received: June 1994)

\begin{abstract}
In this paper, we introduce a Z-graded variant of the periodic cyclic homology of associative algebras which generalizes the infinitesimal cohomology of affine algebras in characteristic 0 and show that it satisfies the Küneth formula (i.e. it commutes with the formation of tensor products). We also show that the Kunneth formula in periodic cyclic homology holds only under the presence of certain finiteness conditions.
\end{abstract}

Key words: Periodic cyclic homology, Künneth formula.

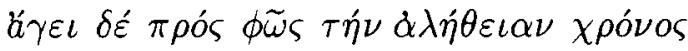

$$
\begin{aligned}
& \text { ( } M \dot{\varepsilon} \nu \alpha \nu \delta \rho o \varsigma, \Gamma \nu \widetilde{\omega} \mu \alpha \iota \mu o \nu o ́ \sigma \tau \iota \chi o \iota, 256)
\end{aligned}
$$

\section{Introduction}

In his investigations of index theory, Connes faced the problem of calculating geometric invariants lying in the $K$-theory of certain noncommutative $\mathbf{C}^{*}$-algebras. In [2], he defined the cyclic cohomology groups $H C^{*}(A)$ of an associative algebra $A$ over the complex numbers, together with a pairing between the $K$-theory $K_{*}(A)$ of $A$ and $H C^{*}(A)$. He showed that if $X$ is a compact manifold, the cyclic homology of the locally convex algebra $A=C^{\infty}(X)$ of smooth functions on $X$ is a certain direct sum of de Rham cohomology groups of $X$. Moreover, in this case, the pairing with $K$-theory recovers the classical Chern character homomorphism of the manifold. Hence, cyclic homology appears as a noncommutative analogue of the de Rham cohomology of a space.

The definition of cyclic homology was extended by Loday and Quillen to algebras over an arbitrary ground ring in [12]. In that paper, the cyclic homology of the algebra of regular functions on a smooth affine variety in characteristic 0 is computed, proving the algebraic version of the above result of Connes.

Connes has also defined the periodic cyclic (co-)homology of an algebra, a 2periodic version of cyclic (co-)homology which is obtained by inverting a certain endomorphism $S$ of the latter. Periodic cyclic homology has a great geometric significance; it is the homotopy invariant part of cyclic homology. According to a theorem due to Feigin and Tsygan (cf. [5]), the periodic cyclic homology of 
the algebra of regular functions on an affine algebraic variety $X$ over a field $k$ of characteristic 0 is isomorphic, as a $\mathbf{Z} / 2 \mathbf{Z}$-graded vector space, to the infinitesimal cohomology of $X$ over Spec $k$, which was defined by Grothendieck in [7] as an analogue of de Rham cohomology for singular varieties, viewed with the even-odd grading.

A disadvantage of periodic cyclic homology compared to infinitesimal cohomology is the fact that the former is $\mathbf{Z} / 2 \mathbf{Z}$-graded, while the latter is $\mathbf{Z}$-graded. It is therefore natural to look for variants of periodic cyclic homology which are intrinsically $\mathbf{Z}$-graded. The periodic cyclic homology $\operatorname{HP}(A)$ of an algebra $A$ comes equipped with a certain decreasing filtration induced by the maps to cyclic homology. The graded object $\mathcal{H}(A)$ associated with this filtration (the formal definition is given in Section 3 ) inherits the homotopy invariance properties of periodic cyclic homology and coincides, when $A$ is an affine algebra in characteristic 0 , with the infinitesimal cohomology of $\operatorname{Spec} A$. In this paper, we study the behavior of the functor $\mathcal{H}$ with respect to tensor products.

For two algebras $A, A^{\prime}$ there are certain obstructions in showing that the cyclic homology $\mathrm{HC}\left(A \otimes A^{\prime}\right)$ is isomorphic to the tensor product $\mathrm{HC}(A) \otimes \operatorname{HC}\left(A^{\prime}\right)$. In order to show that the canonical map (product)

$$
\Theta: \operatorname{HP}(A) \otimes \operatorname{HP}\left(A^{\prime}\right) \rightarrow \operatorname{HP}\left(A \otimes A^{\prime}\right)
$$

is an isomorphism of $\mathbf{Z} / 2 Z$-graded vector spaces, one hopes that these obstructions are unstable and vanish after inverting the endomorphism $S$.

To state our main result, we consider the inverse $\operatorname{limit} \underset{m}{\lim } \mathrm{HC}(A)[-2 m]$ of the system $(\mathrm{HC}(A)[-2 m], S)_{m}$ and its filtration $\mathcal{F}^{A}$ induced by the canonical maps to cyclic homology and similarly for the algebras $A^{\prime}$ and $A \otimes A^{\prime}$. Then, $\underset{m}{\lim } \mathrm{HC}(A)[-2 m], \underset{m}{\lim } \mathrm{HC}\left(A^{\prime}\right)[-2 m]$ and $\underset{\frac{m}{m}}{\lim } \mathrm{HC}\left(A \otimes A^{\prime}\right)[-2 m]$ are complete and Hausdorff with respect to the induced linear topologies. We show that $\underset{m}{\lim } \mathrm{HC}\left(A \otimes A^{\prime}\right)[-2 m]$ can be identified with the completed tensor product $\underset{m}{\lim } \mathrm{HC}(A)[-2 m] \widehat{\otimes} \underset{\frac{m}{m}}{\lim } \mathrm{HC}\left(A^{\prime}\right)[-2 m]$ in such a way that the filtration $\mathcal{F}^{A \otimes A^{\prime}}$ corresponds to the completed tensor product filtration $\mathcal{F}^{A} \widehat{\otimes} \mathcal{F}^{A^{\prime}}$. Hence, passing to the associated graded objects, we obtain an isomorphism

$$
\mathcal{H}\left(A \otimes A^{\prime}\right) \simeq \mathcal{H}(A) \otimes \mathcal{H}\left(A^{\prime}\right)
$$

of $\mathbf{Z}$-graded vector spaces.

In order to lift the above isomorphism to periodic cyclic homology, certain finiteness conditions have to be imposed. In fact, generalizing a result of Kassel [10], we show that the following two conditions are equivalent for an algebra $A$.

(i) The map $\Theta$ is an isomorphism of $\mathbf{Z} / 2 \mathbf{Z}$-graded vector spaces for any algebra $A^{\prime}$.

(ii) The inverse system $(\mathrm{HC}(A)[-2 m], S)_{m}$ satisfies the Mittag-Leffler condition and the periodic cyclic homology $\operatorname{HP}(A)$ is finite dimensional. 
The class of algebras $A$ which satisfy these conditions contains all affine algebras in characteristic 0 and is closed under tensor products.

The contents of this work are as follows. In Section 1, we fix the notations we shall use about supercomplexes and their tensor products. In Section 2, we list certain properties of cotensor products of inverse systems of vector spaces that will be used in the subsequent sections in the special case of the inverse systems associated with the cyclic homology of algebras. In Section 3, we first define the graded functor $\mathcal{H}$ and record some basic properties of it. We then review the relation between cyclic homology and tensor products and prove the Künneth formula for $\mathcal{H}$. Finally, in Section 4, we examine the necessary and sufficient conditions that have to be satisfied for $\Theta$ to be an isomorphism and show that the corresponding class of algebras is closed under tensor products.

\section{NOTATIONS AND TERMINOLOGY}

In this paper, we work over a fixed field $k$. Unless otherwise specified, all vector spaces, algebras and tensor products will be over $k$ and all inverse systems $\mathcal{X}$ will be indexed by $\mathbf{N}$ and consist of $(k$-)vector spaces. If $(C, \partial)$ is a chain complex and $m \in \mathbf{Z}$ we denote by $(C[m], \partial[m])$ the chain complex which is given by $(C[m])_{n}=C_{n-m}$ and $(\partial[m])_{n}=(-1)^{m} \partial_{n-m}$. The same notation will be used to denote an inverse system or a graded vector space with degrees shifted by $m$. The image of an integer $n$ in $\mathbf{Z} / 2 \mathbf{Z}$ (i.e. the parity of $n$ ) will be denoted by $[n]$. Finally, by a decreasing filtration $\mathcal{F}=\left(F_{m}\right)_{m}$ of a vector space $F$ we understand a sequence of subspaces $F \supseteq F_{0} \supseteq F_{1} \supseteq F_{2} \supseteq \cdots$; if $n<0$ we set $F_{n}=F$.

\section{Supercomplexes}

A supercomplex is a $\mathbf{Z} / 2 \mathbf{Z}$-graded vector space $X=X_{0} \oplus X_{1}$ endowed with linear maps $\partial_{0}: X_{0} \rightarrow X_{1}$ and $\partial_{1}: X_{1} \rightarrow X_{0}$ such that $\partial_{0} \partial_{1}=0$ and $\partial_{1} \partial_{0}=0$. Its homology is the $\mathbf{Z} / 2 \mathbf{Z}$-graded vector space $H(X)$ given by

$$
H_{0}(X)=\operatorname{ker} \partial_{0} / \operatorname{im} \partial_{1} \quad \text { and } \quad H_{1}(X)=\operatorname{ker} \partial_{1} / \operatorname{im} \partial_{0}
$$

EXAMPLES 1.1. (i) If $(X, \partial)$ is a chain complex the product complex $\prod_{m} X[-2 m]$ can be viewed as a supercomplex consisting of $\prod_{m} X_{2 m}$ and $\prod_{m} X_{2 m+1}$ in degrees 0 and 1 , respectively. If $(X, \partial)$ is endowed with a chain map $S: X \rightarrow X[2]$, the inverse $\operatorname{limit} \underset{m}{\lim } X[-2 m]$ of the system $(X[-2 m], S)_{m}$ is also a supercomplex; it is the kernel of the endomorphism $1-S$ of $\prod_{m} X[-2 m]$.

(ii) If $(C, b, B)$ is a mixed complex (cf. $[1,10])$ the inverse $\operatorname{limit} \lim \mathcal{B}(C)[-2 m]$ of the system $(\mathcal{B}(C)[-2 m], S)_{m}$ is the supercomplex $\left(\prod_{m} C[-2 m], b+B\right)$. 
Let $X=X_{0} \oplus X_{1}$ and $Y=Y_{0} \oplus Y_{1}$ be $\mathbf{Z} / 2 Z$-graded vector spaces. We shall denote by $X \dot{\otimes} Y$ the $\mathbf{Z} / 2 \mathbf{Z}$-graded vector space which is given by

$$
(X \dot{\otimes} Y)_{0}=\left(X_{0} \otimes Y_{0}\right) \oplus\left(X_{1} \otimes Y_{1}\right)
$$

and

$$
(X \dot{\otimes} Y)_{1}=\left(X_{0} \otimes Y_{1}\right) \oplus\left(X_{1} \otimes Y_{0}\right) .
$$

More generally, if $x_{-}$is a bifunctor of vector spaces, we shall denote by $\dot{x}_{-}$its extension to the $\mathbf{Z} / 2 \mathbf{Z}$-graded case which is defined by formally replacing ' $\otimes$ ' by ' $x$ ' in (1).

If $\left(X, \partial_{0}^{X}, \partial_{1}^{X}\right)$ and $\left(Y, \partial_{0}^{Y}, \partial_{1}^{Y}\right)$ are supercomplexes, the $\mathbf{Z} / 2 \mathbf{Z}$-graded vector space $X \dot{\otimes} Y$ itself becomes a supercomplex if we define $\partial_{0}^{X \dot{\otimes} Y}$ and $\partial_{1}^{X \dot{\otimes} Y}$ by

$$
\partial_{0}^{X \dot{\otimes} Y}=\left(\begin{array}{cc}
1 \otimes \partial_{0}^{Y} & \partial_{1}^{X} \otimes 1 \\
\partial_{0}^{X} \otimes 1 & -1 \otimes \partial_{1}^{Y}
\end{array}\right) \quad \text { and } \quad \partial_{1}^{X \dot{\otimes} Y}=\left(\begin{array}{cc}
1 \otimes \partial_{1}^{Y} & \partial_{1}^{X} \otimes 1 \\
\partial_{0}^{X} \otimes 1 & -1 \otimes \partial_{0}^{Y}
\end{array}\right)
$$

Remarks 1.2. (i) A short exact sequence $0 \rightarrow X^{\prime} \rightarrow X \rightarrow X^{\prime \prime} \rightarrow 0$ of supercomplexes induces a cyclic exact sequence

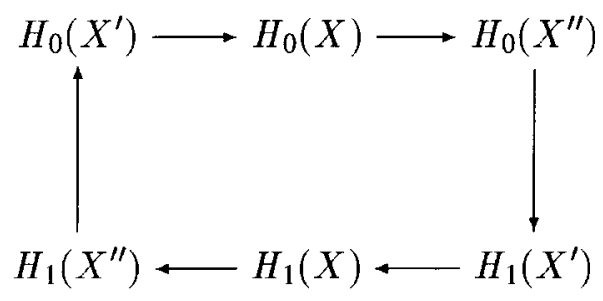

(ii) If $X, Y$ are supercomplexes, there is an identification of $\mathbf{Z} / 2 \mathbf{Z}$-graded vector spaces $H(X \dot{\otimes} Y)=H(X) \dot{\otimes} H(Y)$. This can be proved along the lines of the proof of the usual Künneth formula for the tensor product of two chain complexes, as given, for example, in [8].

\section{Cotensor Products of Inverse Systems}

Let $\mathcal{X}=\left(X_{m}\right)_{m}$ and $\mathcal{X}^{\prime}=\left(X_{m}^{\prime}\right)_{m}$ be two inverse systems whose structural morphisms are denoted by $S$ and $S^{\prime}$. We extend the grading of $\mathcal{X}$ and $\mathcal{X}^{\prime}$ by 0 in negative degrees and consider the inverse system $\mathcal{X} \oslash \mathcal{X}^{\prime}=\prod_{m \geqslant 0}\left(\mathcal{X}[m] \otimes X_{m}^{\prime}\right)$ consisting of $\left(X \otimes X^{\prime}\right)_{n}=\bigoplus_{p+q=n} X_{p} \otimes X_{q}^{\prime}$ in degree $n$ with structural morphisms given by $S \otimes 1$. We also consider the morphism $S \otimes 1-1 \otimes S^{\prime}: \mathcal{X} \oslash \mathcal{X}^{\prime} \rightarrow\left(\mathcal{X} \oslash \mathcal{X}^{\prime}\right)$ [1]; its kernel (resp. cokernel) is the cotensor (resp. cotor) product $\mathcal{X} \square \mathcal{X}^{\prime}=\left(\left(X \square X^{\prime}\right)_{m}\right)_{m}$ $\left(\right.$ resp. $\left.\operatorname{Cotor}\left(\mathcal{X}, \mathcal{X}^{\prime}\right)[1]=\left(\operatorname{Cotor}\left(X, X^{\prime}\right)_{m-1}\right)_{m}\right)$ of $\mathcal{X}$ and $\mathcal{X}^{\prime}$. We note that $\mathcal{X}$ and $\mathcal{X}^{\prime}$ play a symmetric role in $\mathcal{X} \square \mathcal{X}^{\prime}$ and $\operatorname{Cotor}\left(\mathcal{X}, \mathcal{X}^{\prime}\right)$. 
LEMMA 2.1. If $\mathcal{X}=\left(X_{m}\right)_{m}, \mathcal{X}^{\prime}=\left(X_{m}^{\prime}\right)_{m}$ are two inverse systems, then

(i) The inverse system $\operatorname{Cotor}\left(\mathcal{X}, \mathcal{X}^{\prime}\right)$ is essentially zero.

(ii) There is a 6-term exact sequence

$$
\begin{aligned}
& 0 \rightarrow \underset{\lim }{\longleftarrow}\left(\mathcal{X} \square \mathcal{X}^{\prime}\right) \rightarrow \prod_{m \geqslant 0} \underset{\lim }{\longleftarrow}\left(\mathcal{X} \otimes X_{m}^{\prime}\right) \stackrel{1-S^{\prime}}{\longrightarrow} \prod_{m \geqslant 0} \underline{\lim }\left(\mathcal{X} \otimes X_{m}^{\prime}\right) \rightarrow \\
& \rightarrow \underline{\lim }^{1}\left(\mathcal{X} \square \mathcal{X}^{\prime}\right) \rightarrow \prod_{m \geqslant 0} \lim ^{1}\left(\mathcal{X} \otimes X_{m}^{\prime}\right) \stackrel{1-S^{\prime}}{\longrightarrow} \prod_{m \geqslant 0} \lim ^{1}\left(\mathcal{X} \otimes X_{m}^{\prime}\right) \rightarrow 0 .
\end{aligned}
$$

Proof. (i) We show that the map $\operatorname{Cotor}\left(X, X^{\prime}\right)_{m+M} \rightarrow \operatorname{Cotor}\left(X, X^{\prime}\right)_{m}$ is zero if $M>m$. Indeed, let $x_{p} \in X_{p}$ and $x_{q}^{\prime} \in X_{q}^{\prime}$ with $p+q=m+M$. Then, the elements $S^{M} x_{p} \otimes x_{q}^{\prime}$ and $x_{p} \otimes S^{M} x_{q}^{\prime}$ are equivalent modulo the image of $S \otimes 1-1 \otimes S^{\prime}$. In fact, they represent $0 \in \operatorname{Cotor}\left(X, X^{\prime}\right)_{m}$, since we have $p<M$ or $q<M$ and, hence, $S^{M} x_{p} \in X_{p-M}=0$ or $S^{M} x_{q}^{\prime} \in X_{q-M}^{\prime}=0$.

(ii) Let $\mathcal{Y}$ be the image of $S \otimes 1-1 \otimes S^{\prime}: \mathcal{X} \oslash \mathcal{X}^{\prime} \rightarrow\left(\mathcal{X} \oslash \mathcal{X}^{\prime}\right)$ [1]. Then, (i) implies that $\lim ^{i} \mathcal{Y} \simeq \lim ^{i}\left(\mathcal{X} \oslash \mathcal{X}^{\prime}\right), i=0,1$. Therefore, (2) is induced by the short exact sequence of inverse systems $0 \rightarrow \mathcal{X} \square \mathcal{X}^{\prime} \rightarrow \mathcal{X} \oslash \mathcal{X}^{\prime} \rightarrow \mathcal{Y} \rightarrow 0$.

Remark 2.2. One can interpret the 6-term exact sequence (2) as the degeneracy of a certain spectral sequence of the composite functor.

Let us recall that an inverse system $\mathcal{X}=\left(X_{m}\right)_{m}$ is said to satisfy the MittagLeffler condition if for all $n$ the decreasing filtration of $X_{n}$ by the images of $X_{m}$, $m \geqslant n$, is eventually constant.

PROPOSITION 2.3. Let $\mathcal{X}=\left(X_{m}\right)_{m}$ and $\mathcal{X}^{\prime}=\left(X_{m}^{\prime}\right)_{m}$ be two inverse systems satisfying the Mittag-Leffler condition. Then the same is true for their cotensor product $\mathcal{X} \square \mathcal{X}^{\prime}$.

Proof. Consider the inverse system $\left(\mathcal{X} \square \mathcal{X}^{\prime}\right) \otimes E=\mathcal{X} \square\left(\mathcal{X}^{\prime} \otimes E\right)$, where $E$ is an arbitrary vector space. Since $\mathcal{X}$ satisfies the Mittag-Leffler condition, the same is true for the system $\mathcal{X} \otimes X_{m}^{\prime} \otimes E$ for all $m$. Hence, (2) shows that there is an exact sequence

$$
\begin{aligned}
& \prod_{m \geqslant 0} \lim _{\leftarrow}\left(\mathcal{X} \otimes X_{m}^{\prime} \otimes E\right) \stackrel{1-S^{\prime}}{\longrightarrow} \prod_{m \geqslant 0} \underset{\lim }{\longleftarrow}\left(\mathcal{X} \otimes X_{m}^{\prime} \otimes E\right) \\
& \quad \rightarrow \underline{\lim }^{1}\left(\mathcal{X} \square \mathcal{X}^{\prime} \otimes E\right) \rightarrow 0 .
\end{aligned}
$$

Since $\mathcal{X}^{\prime}$ satisfies the Mittag-Leffler condition, one can show that the same is true for the system $\left(\lim \left(\mathcal{X} \otimes X_{m}^{\prime} \otimes E\right)\right)_{m}$. Therefore, the map $1-S^{\prime}$ in the exact sequence above is surjective and, hence, $\lim ^{1}\left(\mathcal{X} \square \mathcal{X}^{\prime} \otimes E\right)=0$. The result follows using $[4$, Corollary 6]. 
Remark 2.4. If $k$ is any ring and $\mathcal{X}$ (resp. $\mathcal{X}^{\prime}$ ) an inverse system of right (resp. left) $k$-modules, one can define as above the inverse systems of Abelian groups $\mathcal{X} \oslash \mathcal{X}^{\prime}, \mathcal{X} \square \mathcal{X}^{\prime}$ and $\operatorname{Cotor}\left(\mathcal{X}, \mathcal{X}^{\prime}\right)$ and prove Lemma 2.1. The result of Proposition 2.3 generalizes as well, provided that we require at least one of the systems $\mathcal{X}, \mathcal{X}^{\prime}$ to consist of flat modules. Indeed, if $\mathcal{X}=\left(X_{m}\right)_{m}$ satisfies the Mittag-Leffler condition and $X_{m}$ is flat for all $m$ the functor $\lim \left(\mathcal{X} \otimes_{-}\right)$of left $k$-modules is exact and, hence, the above proof is still valid, if we choose $E$ to be a free Abelian group of infinite rank.

If $\mathcal{X}=\left(X_{m}\right)_{m}$ is an inverse system and $n \in N$ we shall denote by $\sigma_{n}^{\mathcal{X}}$ the canonical map $\lim _{\longleftarrow} \mathcal{X} \rightarrow X_{n}$. One can verify that $\operatorname{im} \sigma_{n}^{\mathcal{X} \otimes E}=\operatorname{im} \sigma_{n}^{\mathcal{X}} \otimes E$ for any vector space $E$.

If $\mathcal{X}=\left(X_{m}\right)_{m}, \mathcal{X}^{\prime}=\left(X_{m}^{\prime}\right)_{m}$ are two inverse systems, we shall consider the map

$$
\tau^{\mathcal{X}, \mathcal{X}^{\prime}}:\left(\lim ^{\operatorname{X}}\right) \otimes\left(\lim ^{\mathcal{X}^{\prime}}\right) \rightarrow \underset{\lim }{\longleftarrow}\left(\mathcal{X} \square \mathcal{X}^{\prime}\right)
$$

which, when followed by the composition

$$
\underline{\lim }\left(\mathcal{X} \square \mathcal{X}^{\prime}\right) \stackrel{\sigma_{n}^{\mathcal{X}} \square \mathcal{X}^{\prime}}{\longrightarrow} \quad\left(X \square X^{\prime}\right)_{n} \hookrightarrow\left(X \otimes X^{\prime}\right)_{n} \rightarrow X_{p} \otimes X_{q}^{\prime},
$$

coincides with $\sigma_{p}^{\mathcal{X}} \otimes \sigma_{q}^{\mathcal{X}^{\prime}}$ for all $p, q, n$ with $p+q=n$.

PROPOSITION 2.5. If $\mathcal{X}=\left(X_{m}\right)_{m}, \mathcal{X}^{\prime}=\left(X_{m}^{\prime}\right)_{m}$ are two inverse systems, then

(i) $\tau^{\mathcal{X}, \mathcal{X}^{\prime}}$ is injective.

(ii) $\operatorname{ker} \varrho_{n}^{\mathcal{X}, \mathcal{X}^{\prime}}=\sum_{p+q=n-1} \operatorname{ker} \sigma_{p}^{\mathcal{X}} \otimes \operatorname{ker} \sigma_{q}^{\mathcal{X}^{\prime}}$, where $\varrho_{n}^{\mathcal{X}, \mathcal{X}^{\prime}}=\sigma_{n}^{\mathcal{X} \square \mathcal{X}^{\prime}} \tau^{\mathcal{X}, \mathcal{X}^{\prime}}$.

(iii) $\operatorname{im} \varrho_{n}^{\mathcal{X}, \mathcal{X}^{\prime}}=\operatorname{im} \sigma_{n}^{\mathcal{X} \square \mathcal{X}^{\prime}}$.

(iv) $\tau^{\mathcal{X}, \mathcal{X}^{\prime}}$ induces an isomorphism

$$
\begin{aligned}
\operatorname{ker} \sigma_{n-1}^{\mathcal{X} \square \mathcal{X}^{\prime}} / \operatorname{ker} \sigma_{n}^{\mathcal{X} \square \mathcal{X}^{\prime}} & \simeq \operatorname{ker} \varrho_{n-1}^{\mathcal{X}, \mathcal{X}^{\prime}} / \operatorname{ker} \varrho_{n}^{\mathcal{X}, \mathcal{X}^{\prime}} \\
& \simeq \bigoplus_{p+q=n}\left(\operatorname{ker} \sigma_{p-1}^{\mathcal{X}} / \operatorname{ker} \sigma_{p}^{\mathcal{X}}\right) \otimes\left(\operatorname{ker} \sigma_{q-1}^{\mathcal{X}^{\prime}} / \operatorname{ker} \sigma_{q}^{\mathcal{X}^{\prime}}\right) .
\end{aligned}
$$

Proof. (i) Using the notations of [4] and the exact sequence (2), $\tau^{\mathcal{X}, \mathcal{X}^{\prime}}$ is the composition

$$
\begin{aligned}
& (\stackrel{\lim }{\longleftarrow}) \otimes\left(\stackrel{\lim }{\longleftarrow} \mathcal{X}^{\prime}\right) \stackrel{\Phi^{0}}{\longrightarrow} \underset{m}{\lim }\left(\left(\stackrel{\lim \mathcal{X}}{\longleftarrow} \otimes X_{m}^{\prime}\right) \stackrel{\Phi^{0}}{\longrightarrow} \underset{m}{\lim }\left(\stackrel{\lim }{\longleftarrow}\left(\mathcal{X} \otimes X_{m}^{\prime}\right)\right)\right. \\
& =\lim _{\left(\mathcal{X} \square \mathcal{X}^{\prime}\right)}
\end{aligned}
$$

Therefore, the injectivity of $\tau^{\mathcal{X}, \mathcal{X}^{\prime}}$ follows from [4, Remark 1(i)]. 
(ii) Since $\varrho_{n}^{\mathcal{X}, \mathcal{X}^{\prime}}$ followed by the composition $\left(X \square X^{\prime}\right)_{n} \hookrightarrow\left(X \otimes X^{\prime}\right)_{n} \rightarrow X_{p} \otimes$ $X_{q}^{\prime}$ is the map $\sigma_{p}^{\mathcal{X}} \otimes \sigma_{q}^{\mathcal{X}^{\prime \prime}}$ for all $p, q$ with $p+q=n$, we have

$$
\begin{aligned}
\operatorname{ker} \varrho_{n}^{\mathcal{X}, \mathcal{X}^{\prime}} & =\bigcap_{p+q=n} \operatorname{ker}\left(\sigma_{p}^{\mathcal{X}} \otimes \sigma_{q}^{\mathcal{X}^{\prime}}\right) \\
& =\bigcap_{p+q=n}\left(\operatorname{ker} \sigma_{p}^{\mathcal{X}} \otimes \underline{\lim } \mathcal{X}^{\prime}+\underline{\left.\lim \mathcal{X} \otimes \operatorname{ker} \sigma_{q}^{\mathcal{X}^{\prime}}\right)}\right. \\
& =\sum_{p+q=n-1} \operatorname{ker} \sigma_{p}^{\mathcal{X}} \otimes \operatorname{ker}{\sigma_{q}^{\mathcal{X}^{\prime}}}
\end{aligned}
$$

where the last equality follows from the next lemma.

LEMMA 2.6. Let $F, F^{\prime}$ be vector spaces endowed with decreasing filtrations $\mathcal{F}=\left(F_{m}\right)_{m}$ and $\mathcal{F}^{\prime}=\left(F_{m}^{\prime}\right)_{m}$, respectively. Then,

$$
\bigcap_{p+q=n}\left(F_{p} \otimes F^{\prime}+F \otimes F_{q}^{\prime}\right)=\sum_{p+q=n-1} F_{p} \otimes F_{q}^{\prime}
$$

(iii) An element of $\lim \left(\mathcal{X} \square \mathcal{X}^{\prime}\right)$ is a double family

$$
\Xi=\left(\xi_{p, q}\right)_{p, q} \in \prod_{p, q}\left(X_{p} \otimes X_{q}^{\prime}\right)
$$

with

$$
(S \otimes 1) \xi_{p+1, q}=\xi_{p, q}=\left(1 \otimes S^{\prime}\right) \xi_{p, q+1}
$$

for all $p, q$. In particular,

$$
\begin{aligned}
& \xi_{p, q} \in \operatorname{im} \sigma_{p}^{\mathcal{X} \otimes X_{q}^{\prime}} \bigcap \operatorname{im} \sigma_{q}^{X} \otimes \mathcal{X}^{\prime} \\
& \quad=\left(\operatorname{im} \sigma_{p}^{\mathcal{X}} \otimes X_{q}^{\prime}\right) \bigcap\left(X_{p} \otimes \operatorname{im}{\sigma_{q}^{\mathcal{X}^{\prime}}}^{X^{\prime}}\right)=\operatorname{im}{\sigma_{p}^{\mathcal{X}} \otimes \operatorname{im}{\sigma_{q}^{\mathcal{X}^{\prime}}}^{\prime}}
\end{aligned}
$$

for all $p, q$. Since $\sigma_{n}^{\mathcal{X} \square \mathcal{X}^{\prime}}(\Xi)=\left(\xi_{p, q}\right)_{p+q=n}$ is determined by $\xi_{n, n} \in \operatorname{im} \sigma_{n}^{\mathcal{X}} \otimes$ $\operatorname{im} \sigma_{n}^{\mathcal{X}^{\prime}}$, we conclude that $\sigma_{n}^{\mathcal{X}} \square \mathcal{X}^{\prime}(\Xi)$ belongs in the image of $\varrho_{n}^{\mathcal{X}, \mathcal{X}^{\prime}}$.

(iv) This is a consequence of (ii) and (iii).

Remarks 2.7. (i) If $\mathcal{F}=\left(F_{m}\right)_{m}, \mathcal{F}^{\prime}=\left(F_{m}^{\prime}\right)_{m}$ are decreasing filtrations of the vector spaces $F$ and $F^{\prime}$, respectively, the tensor product filtration $\mathcal{F} \otimes \mathcal{F}^{\prime}=$ $\left(\left(F \otimes F^{\prime}\right)_{m}\right)_{m}$ of $F \otimes F^{\prime}$ is defined by $\left(F \otimes F^{\prime}\right)_{m}=\sum_{p+q=m} F_{p} \otimes F_{q}^{\prime}$. The induced linear topology on $F \otimes F^{\prime}$ is the tensor product topology of the corresponding linear topologies on $F$ and $F^{\prime}$; let $F \hat{\otimes} F^{\prime}$ be the completion of $F \otimes F^{\prime}$ with respect to 
that topology. If the filtrations $\mathcal{F}, \mathcal{F}^{\prime}$ are Hausdorff, the same is true for $\mathcal{F} \otimes \mathcal{F}^{\prime}$ and, hence, the canonical map

$$
F \otimes F^{\prime} \rightarrow F \hat{\otimes} F^{\prime}
$$

is injective. It need not be an isomorphism even if both $F$ and $F^{\prime}$ are complete. Let $\mathcal{F} \widehat{\otimes} \mathcal{F}^{\prime}$ be the filtration of $F \widehat{\otimes} F^{\prime}$ obtained by completing $\mathcal{F} \otimes \mathcal{F}^{\prime}$. Then, passing to the associated graded objects, one obtains an isomorphism

$$
\mathrm{gr}^{\mathcal{F} \widehat{\otimes} \mathcal{F}^{\prime}}\left(F \hat{\otimes} F^{\prime}\right) \simeq \operatorname{gr}^{\mathcal{F} \otimes \mathcal{F}^{\prime}}\left(F \otimes F^{\prime}\right) \simeq \operatorname{gr}^{\mathcal{F}}(F) \otimes \operatorname{gr}^{\mathcal{F}^{\prime}}\left(F^{\prime}\right)
$$

of graded vector spaces.

For an inverse system $\mathcal{X}$ we consider the decreasing filtration $\mathcal{F}^{\mathcal{X}}=\left(\operatorname{ker} \sigma_{m}^{\mathcal{X}}\right)_{m}$ of $\lim \mathcal{X}$. If $\mathcal{X}^{\prime}$ is another such system assertion (ii) of Proposition 2.5 shows that the tensor product filtration $\mathcal{F}^{\mathcal{X}} \otimes \mathcal{F}^{\mathcal{X}^{\prime}}$ of $(\lim \mathcal{X}) \otimes\left(\lim \mathcal{X}^{\prime}\right)$ is given up to a degree shift by the kernels of the various maps $\varrho^{\mathcal{X}}, \mathcal{X}^{\prime \prime}$. Using assertion (iii), we can identify $\lim \left(\mathcal{X} \square \mathcal{X}^{\prime}\right)$ with the completion $(\lim \mathcal{X}) \otimes\left(\lim \mathcal{X}^{\prime}\right)$ in such a way that

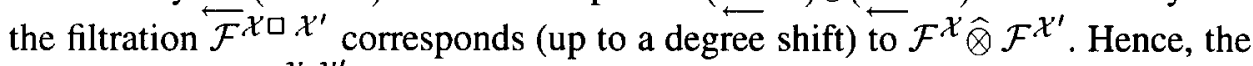
monomorphism $\tau^{\mathcal{X}, \mathcal{X}^{\prime}}$ is a special case of (4) and the isomorphism of assertion (iv) of Proposition 2.5 a special case of (5).

(ii) Suppose that $\tau^{\mathcal{X}, \mathcal{X}^{\prime}}$ is an isomorphism and $\operatorname{ker}{\sigma_{n}^{\mathcal{X}^{\prime}}}^{\prime} \neq 0$ for all $n$. Then, the decomposition (3) of $\tau^{\mathcal{X}, \mathcal{X}^{\prime}}$ and [4, Proposition 2] show that $\lim \mathcal{X}$ is finitedimensional. In fact, one can show that $\tau^{\mathcal{X}, \mathcal{X}^{\prime \prime}}$ is an isomorphism if and only if one of the following conditions is satisfied:

( $\alpha) \lim \mathcal{X}$ is finite-dimensional.

$\left(\alpha^{\prime}\right) \lim \mathcal{X}^{\prime}$ is finite-dimensional.

$(\beta)$ The canonical maps $\sigma_{n}^{\mathcal{X}}$ and $\sigma_{n}^{\mathcal{X}^{\prime}}$ are injective for $n \gg 0$.

\section{The Graded Functor $\mathcal{H}$}

Let $A$ be an algebra. The reader can find all the basic definitions and results about the cyclic and periodic cyclic homology $\mathrm{HC}(A)$ and $\mathrm{HP}(A)$ of $A$ in Loday's book [11]. Cyclic homology comes equipped with a certain endomorphism $S$ of degree -2 . The relation between cyclic and periodic cyclic homology can be expressed in terms of the inverse system $(\mathrm{HC}(A)[-2 m], S)_{m}$ by the short exact sequence

$$
0 \rightarrow \underset{m}{\lim _{m}^{1}} \mathrm{HC}(A)[-2 m-1] \rightarrow \mathrm{HP}(A) \rightarrow \underset{m}{\lim } \mathrm{HC}(A)[-2 m] \rightarrow 0 .
$$

Let $n \in \mathbf{Z}, *=[n]$ and consider the map $\bar{\sigma}_{n}^{A}: \mathrm{HP}_{*}(A) \rightarrow \mathrm{HC}_{n}(A)$ defined as the composition of the surjection $\mathrm{HP}_{*}(A) \rightarrow \underset{m}{\lim } \mathrm{HC}_{*+2 m}(A)$ followed by the structural morphism $\sigma_{n}^{A}: \underset{m}{\lim } \mathrm{HC}_{*+2 m}(A) \rightarrow \mathrm{HC}_{n}(A)$ of the inverse limit $\underset{m}{\lim } \mathrm{CC}_{*+2 m}(A)$. We consider the decreasing filtration of $\operatorname{HP}_{*}(A)$ by its subspaces $\operatorname{ker} \bar{\sigma}_{n}^{A},[n]=*$, and 
note that the induced linear topology is Hausdorff if and only if $\lim ^{1} \mathrm{HC}_{*+2 m+1}(A)=$ 0 . We define

$$
\mathcal{H}_{n}(A)=\operatorname{ker} \bar{\sigma}_{n-2}^{A} / \operatorname{ker} \bar{\sigma}_{n}^{A}=\operatorname{ker} \sigma_{n-2}^{A} / \operatorname{ker} \sigma_{n}^{A} .
$$

In particular, $\mathcal{H}_{n}(A)=0$ if $n<0$. We note that there is an identification

$$
\begin{aligned}
& \mathcal{H}_{n}(A) \\
& \quad \simeq \operatorname{im}\left(\operatorname{HP}_{*}(A) \stackrel{\bar{\sigma}_{n}^{A}}{\longrightarrow} \mathrm{HC}_{n}(A)\right) \bigcap \operatorname{ker}\left(\mathrm{HC}_{n}(A) \stackrel{S}{\longrightarrow} \mathrm{HC}_{n-2}(A)\right) \\
& \quad=\operatorname{im}\left(\underset{\frac{m}{\lim }}{\operatorname{loc}} \mathrm{HC}_{*+2 m}(A) \stackrel{\sigma_{n}^{A}}{\longrightarrow} \mathrm{HC}_{n}(A)\right) \bigcap \operatorname{ker}\left(\mathrm{HC}_{n}(A) \stackrel{S}{\longrightarrow} \mathrm{HC}_{n-2}(A)\right) .
\end{aligned}
$$

Remarks 3.1. (i) The graded functor $\mathcal{H}=\bigoplus_{n} \mathcal{H}_{n}$ has the following properties:

Base change. There is an identification $\mathcal{H}\left(A^{K}\right)=\mathcal{H}(A) \otimes K$ for any field extension $K / k$, where we denote by $A^{K}$ the $K$-algebra $A \otimes K$. This follows since $\operatorname{im} \sigma_{n}^{A \otimes K}=\operatorname{im} \sigma_{n}^{A} \otimes K$.

Homotopy invariance. If char $k=0$ and $A=A_{0} \oplus A_{1} \oplus A_{2} \oplus \cdots$ is a graded algebra, the inclusion $A_{0} \hookrightarrow A$ induces an isomorphism $\mathcal{H}\left(A_{0}\right) \simeq \mathcal{H}(A)$. This is a consequence of the corresponding property of limHC $[-2 m]$, which is itself proved using Goodwillie's theorem on the action of derivations in cyclic homology (cf. [6]), applied to the special case of the Euler field acting on $A$ (cf. [13, Proposition 2.4]).

Affine algebras. If char $k=0$ and $A$ is a commutative algebra of finite type, there is a canonical isomorphism of graded vector spaces $\mathcal{H}(A) \simeq H_{\text {inf }}(\operatorname{Spec} A)$, where $H_{\text {inf }}(\operatorname{Spec} A)$ is the infinitesimal cohomology of Spec $A$ over Spec $k$ introduced by Grothendieck in [7]. This is a consequence of the computation of $\operatorname{HP}(A)$, which is due to Feigin and Tsygan (cf. [5]), combined with a result of Deligne, Bloom and Herrera, which establishes the embedding of the infinitesimal cohomology into the de Rham (Kähler) cohomology over $k=\mathrm{C}$ (cf. [3]).

(ii) If the inverse system $(\mathrm{HC}(A)[-2 m], S)_{m}$ satisfies the Mittag-Leffler condition, the graded vector space $\mathcal{H}(A)=\bigoplus_{n} \mathcal{H}_{n}(A)$ is isomorphic to the limit term $E^{\infty}(A)=\bigoplus_{n} E_{n}^{\infty}(A)$ of the spectral sequence associated with Connes' exact couple of $A$ (cf. [3]).

We now examine the behavior of $\mathcal{H}$ with respect to tensor products. Let $A, A^{\prime}$ be two algebras. We shall first review the relation between the cyclic homology groups of $A, A^{\prime}$ and that of $A \otimes A^{\prime}$; for more details, the reader is referred to [1], [9], [10] and [11]. Let $\mathcal{B}(\bar{C}(A)), \mathcal{B}\left(\bar{C}\left(A^{\prime}\right)\right)$ and $\mathcal{B}\left(\bar{C}(A) \otimes \bar{C}\left(A^{\prime}\right)\right)$ be the chain complexes associated with the mixed complexes $(\bar{C}(A), b, B),\left(\bar{C}\left(A^{\prime}\right), b, B\right)$ 
and $\left(\bar{C}(A) \otimes \bar{C}\left(A^{\prime}\right), b, B\right)$, respectively. Here, $(\bar{C}(A), b)$ and $\left(\bar{C}\left(A^{\prime}\right), b\right)$ are the normalized Hochschild complexes of $A$ and $A^{\prime}$, while $B$ is Connes' operator. Then, $\mathcal{B}(\bar{C}(A))=k[u] \otimes \bar{C}(A)$ as graded vector spaces, where the polynomial algebra $k[u]$ is graded with deg $u=2$, and similarly for $\mathcal{B}\left(\bar{C}\left(A^{\prime}\right)\right)$ and $\mathcal{B}\left(\bar{C}(A) \otimes \bar{C}\left(A^{\prime}\right)\right)$. We also consider the degree -2 endomorphisms $S$ of these complexes which are induced by the endomorphism $S$ of $k[u]$ given by $S\left(u^{n}\right)=u^{n-1}$ (resp. 0) if $n>0$ (resp. $n=0$ ).

There is a short exact sequence of complexes

$$
\begin{gathered}
0 \rightarrow \mathcal{B}\left(\bar{C}(A) \otimes \bar{C}\left(A^{\prime}\right)\right) \stackrel{\Delta}{\longrightarrow} \mathcal{B}(\bar{C}(A)) \otimes \mathcal{B}\left(\bar{C}\left(A^{\prime}\right)\right) \\
\stackrel{S \otimes 1-1 \otimes S}{\longrightarrow}\left(\mathcal{B}(\bar{C}(A)) \otimes \mathcal{B}\left(\bar{C}\left(A^{\prime}\right)\right)\right)[2] \rightarrow 0,
\end{gathered}
$$

where $\Delta$ is defined by

$$
u^{t} \otimes\left(\xi \otimes \xi^{\prime}\right) \mapsto \sum_{\alpha+\beta=t}\left(u^{\alpha} \otimes \xi\right) \otimes\left(u^{\beta} \otimes \xi^{\prime}\right)
$$

for all $t \in \mathbf{N}, \xi \in \bar{C}(A)$ and $\xi^{\prime} \in \bar{C}\left(A^{\prime}\right)$. This is a short exact sequence of inverse systems of complexes if we endow $\mathcal{B}(\bar{C}(A)) \otimes \mathcal{B}\left(\bar{C}\left(A^{\prime}\right)\right)$ with the degree -2 endomorphism $S \otimes 1$. There is a functorial quasi-isomorphism $\mathcal{B}(\bar{C}(A) \otimes$ $\left.\bar{C}\left(A^{\prime}\right)\right) \rightarrow \mathcal{B}\left(\bar{C}\left(A \otimes A^{\prime}\right)\right)$ which commutes with $S$ and, hence, the long exact sequence associated with (6) takes the form

$$
\begin{aligned}
\cdots & \rightarrow\left(\mathrm{HC}(A) \otimes \mathrm{HC}\left(A^{\prime}\right)\right)_{n+1} \stackrel{S \otimes 1-1 \otimes S}{\longrightarrow}\left(\mathrm{HC}(A) \otimes \mathrm{HC}\left(A^{\prime}\right)\right)_{n-1} \\
& \rightarrow \mathrm{HC}_{n}\left(A \otimes A^{\prime}\right) \stackrel{\Delta}{\longrightarrow} \\
& \left(\mathrm{HC}(A) \otimes \mathrm{HC}\left(A^{\prime}\right)\right)_{n} \stackrel{S \otimes 1-1 \otimes S}{\longrightarrow}\left(\mathrm{HC}(A) \otimes \mathrm{HC}\left(A^{\prime}\right)\right)_{n-2} \rightarrow \cdots .
\end{aligned}
$$

If we denote by $\left(\mathrm{HC}(A) \square \mathrm{HC}\left(A^{\prime}\right)\right)_{n}$ (resp. $\left.\operatorname{Cotor}\left(\mathrm{HC}(A), \mathrm{HC}\left(A^{\prime}\right)\right)_{n-2}\right)$ the kernel (resp. cokernel) of the map

$$
\left(\mathrm{HC}(A) \otimes \mathrm{HC}\left(A^{\prime}\right)\right)_{n} \stackrel{S \otimes 1-1 \otimes S}{\longrightarrow}\left(\mathrm{HC}(A) \otimes \mathrm{HC}\left(A^{\prime}\right)\right)_{n-2},
$$

then there is a short exact sequence

$$
\begin{aligned}
0 & \rightarrow \operatorname{Cotor}\left(\mathrm{HC}(A), \mathrm{HC}\left(A^{\prime}\right)\right)[1] \\
& \rightarrow \mathrm{HC}\left(A \otimes A^{\prime}\right) \stackrel{\Delta}{\longrightarrow} \mathrm{HC}(A) \square \mathrm{HC}\left(A^{\prime}\right) \rightarrow 0 .
\end{aligned}
$$

This is an exact sequence of inverse systems, where the structural morphisms of the terms involving the cotor and cotensor products are induced by $S \otimes 1$ (or, equivalently, by $1 \otimes S$ ). 
We note that $\lim _{m} \mathcal{B}(\bar{C}(A))[-2 m]=\left(\prod_{m} \bar{C}(A)[-2 m], b+B\right)$ (Example 1.1(ii)) and similarly for $\bar{C}\left(A^{\prime}\right)$ and $\bar{C}(A) \otimes \bar{C}\left(A^{\prime}\right)$. We shall consider the morphism of supercomplexes

$$
\begin{aligned}
& \Theta:\left(\underset{\frac{\lim }{m}}{\mathcal{B}}(\bar{C}(A))[-2 m]\right) \dot{\otimes}\left(\underset{\frac{\lim }{m}}{\mathcal{B}}\left(\bar{C}\left(A^{\prime}\right)\right)[-2 m]\right) \\
& \rightarrow \underset{m}{\lim } \mathcal{B}\left(\bar{C}(A) \otimes \bar{C}\left(A^{\prime}\right)\right)[-2 m]
\end{aligned}
$$

whose restriction $\Theta^{00}$ to chains supported in (even) $\otimes\left(\right.$ even) degrees maps $\left(\xi_{m}\right)_{m} \otimes$ $\left(\xi_{m}^{\prime}\right)_{m}$ onto $\left(\sum_{i+j=m} \xi_{i} \otimes \xi_{j}^{\prime}\right)_{m}$ for any $\left(\xi_{m}\right)_{m} \in \prod_{m} \bar{C}_{2 m}(A)$ and $\left(\xi_{m}^{\prime}\right)_{m} \in$ $\prod_{m} \bar{C}_{2 m}\left(A^{\prime}\right)$ (and similarly for the restrictions $\Theta^{01}, \Theta^{10}$ and $\Theta^{11}$ ).

We denote by $\sigma^{\square}$ the structural morphisms of the inverse limit

$$
\frac{\lim }{m}\left(\mathrm{HC}(A) \square \mathrm{HC}\left(A^{\prime}\right)\right)[-2 m] \text {. }
$$

LEMMA 3.2. If $A, A^{\prime}$ are two algebras, then

(i) $\Delta$ induces an isomorphism

$$
\underset{\frac{\lim }{m}}{\mathrm{HC}}\left(A \otimes A^{\prime}\right)[-2 m] \simeq \underset{\frac{\lim }{m}}{ }\left(\mathrm{HC}(A) \square \mathrm{HC}\left(A^{\prime}\right)\right)[-2 m] .
$$

(ii) There is a canonical monomorphism

$$
\begin{aligned}
\tau: & \left(\lim _{m} \mathrm{HC}(A)[-2 m]\right) \dot{\otimes}\left(\frac{\lim }{m} \mathrm{HC}\left(A^{\prime}\right)[-2 m]\right) \\
& \rightarrow \underset{m}{\lim }\left(\mathrm{HC}(A) \square \mathrm{HC}\left(A^{\prime}\right)\right)[-2 m],
\end{aligned}
$$

such that the composition $\Delta^{-1} \tau$ is induced by $\Theta$.

Proof. (i) Since the inverse system $\left(\operatorname{Cotor}\left(\mathrm{HC}(A), \mathrm{HC}\left(A^{\prime}\right)\right)[-2 m]\right)_{m}$ is essentially zero (Lemma 2.1(i)), the result follows from the short exact sequence (7).

(ii) Let us define $\tau$ such that $\sigma_{n}^{\square} \tau$, followed by the composition

$$
\left(\mathrm{HC}(A) \square \mathrm{HC}\left(A^{\prime}\right)\right)_{n} \hookrightarrow\left(\mathrm{HC}(A) \otimes \mathrm{HC}\left(A^{\prime}\right)\right)_{n} \rightarrow \mathrm{HC}_{p}(A) \otimes \mathrm{HC}_{q}\left(A^{\prime}\right),
$$

is the map $\sigma_{p}^{A} \otimes \sigma_{q}^{A^{\prime}}$ for all $p, q, n$ with $p+q=n$. Then, Proposition 2.5(i) shows that $\tau$ is injective. The last claim of assertion (ii) follows since given any (for example) even chains $\left(\xi_{m}\right)_{m} \in \prod_{m} \bar{C}_{2 m}(A),\left(\xi_{m}^{\prime}\right)_{m} \in \prod_{m} \bar{C}_{2 m}\left(A^{\prime}\right)$ and any $n \in \mathbf{N}$, the element

$$
\begin{aligned}
& \left(\sum_{i+j=n} \xi_{i} \otimes \xi_{j}^{\prime}, \sum_{i+j=n-1} \xi_{i} \otimes \xi_{j}^{\prime}, \ldots, \xi_{0} \otimes \xi_{0}^{\prime}\right) \\
& =\sum_{i+j+t=n} u^{t} \otimes\left(\xi_{i} \otimes \xi_{j}^{\prime}\right)
\end{aligned}
$$


of $\left(\mathcal{B}\left(\bar{C}(A) \otimes \bar{C}\left(A^{\prime}\right)\right)\right)_{2 n}$ maps under $\Delta$ onto the element

$$
\begin{gathered}
\sum_{i+j=n}\left(\xi_{i}, \xi_{i-1}, \ldots, \xi_{0}\right) \otimes\left(\xi_{j}^{\prime}, \xi_{j-1}^{\prime}, \ldots, \xi_{0}^{\prime}\right) \\
=\sum_{i+j+\alpha+\beta=n}\left(u^{\alpha} \otimes \xi_{i}\right) \otimes\left(u^{\beta} \otimes \xi_{j}^{\prime}\right)
\end{gathered}
$$

of $\left(\mathcal{B}(\bar{C}(A)) \otimes \mathcal{B}\left(\bar{C}\left(A^{\prime}\right)\right)\right)_{2 n}$.

THEOREM 3.3. For any two algebras $A, A^{\prime}$, the comultiplication $\Delta$ induces an isomorphism $\mathcal{H}\left(A \otimes A^{\prime}\right) \simeq \mathcal{H}(A) \otimes \mathcal{H}\left(A^{\prime}\right)$ of graded vector spaces.

Proof. Given $n \in \mathbf{N}$, we let $*=[n]$ and consider the following diagram

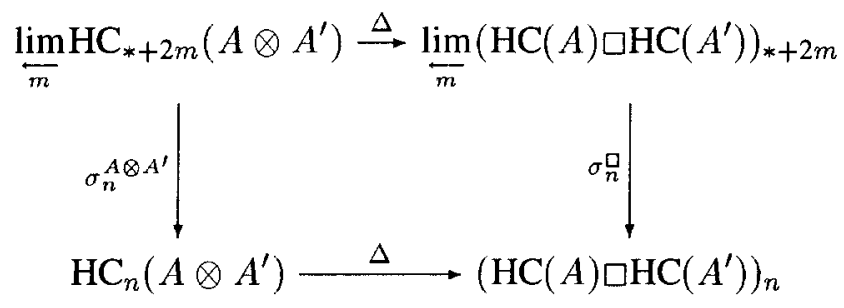

where the top arrow is the isomorphism of Lemma 3.2(i). We also consider $\varrho_{n}=$ $\sigma_{n}^{\square} \tau$, where $\tau$ is the monomorphism of Lemma 3.2(ii), and the graded vector spaces $H^{\square}=\bigoplus_{n} H_{n}^{\square}$ and $H^{\ell}=\bigoplus_{n} H_{n}^{\varrho}$ which are defined by $H_{n}^{\square}=\operatorname{ker} \sigma_{n-2}^{\square} / \operatorname{ker} \sigma_{n}^{\square}$ and $H_{n}^{\varrho}=\operatorname{ker} \varrho_{n-2} / \operatorname{ker} \varrho_{n}$. We shall prove that

(i) $\operatorname{ker} \varrho_{n}=\sum_{p+q=n-2} \operatorname{ker} \sigma_{p}^{A} \otimes \operatorname{ker} \sigma_{q}^{A^{\prime}}$,

(ii) $\tau$ induces an identification $H^{\varrho}=H^{\square}$,

(iii) $\tau\left(\operatorname{ker} \varrho_{n}\right) \subseteq \Delta\left(\operatorname{ker} \sigma_{n}^{A \otimes A^{\prime}}\right) \subseteq \operatorname{ker} \sigma_{n}^{\square}$ and

(iv) $\Delta: \mathcal{H}\left(A \otimes A^{\prime}\right) \rightarrow H^{\square}$ is injective.

Suppose that these assertions are proved. Then, we conclude the proof of the theorem as follows. Assertions (i) and (ii) imply that

$$
\begin{aligned}
H_{n}^{\square}=H_{n}^{\varrho} & =\bigoplus_{p+q=n}\left(\operatorname{ker} \sigma_{p-2}^{A} / \operatorname{ker} \sigma_{p}^{A}\right) \otimes\left(\operatorname{ker} \sigma_{q-2}^{A^{\prime}} / \operatorname{ker} \sigma_{q}^{A^{\prime}}\right) \\
& =\bigoplus_{p+q=n} \mathcal{H}_{p}(A) \otimes \mathcal{H}_{q}\left(A^{\prime}\right) \\
& =\left(\mathcal{H}(A) \otimes \mathcal{H}\left(A^{\prime}\right)\right)_{n} .
\end{aligned}
$$

It follows from (ii) and (iii) that $\Delta: \mathcal{H}\left(A \otimes A^{\prime}\right) \rightarrow H^{\square}$ admits a right inverse, namely the map $\Delta^{-1} \tau: H^{\varrho} \rightarrow \mathcal{H}\left(A \otimes A^{\prime}\right)$. Hence, (iv) implies that $\Delta: \mathcal{H}(A \otimes$ $\left.A^{\prime}\right) \rightarrow H^{\square}$ is an isomorphism. 
Proof of assertion (i). Suppose that $n=2 l$ is even and consider the restriction $\varrho_{n}^{00}$ of $\varrho_{n}$ on

$$
\left(\frac{\lim }{m} H C_{2 m}(A)\right) \otimes\left(\underset{\frac{\lim }{m}}{\operatorname{lic}} \mathrm{C}_{2 m}\left(A^{\prime}\right)\right) .
$$

Then,

$$
\operatorname{ker} \varrho_{n}^{00}=\sum_{p+q=l-1} \operatorname{ker} \sigma_{2 p}^{A} \otimes \operatorname{ker} \sigma_{2 q}^{A^{\prime}}
$$

as shown in Proposition 2.5(ii). Similarly,

$$
\operatorname{ker} \varrho_{n}^{11}=\sum_{p+q=l-1} \operatorname{ker} \sigma_{2 p+1}^{A} \otimes \operatorname{ker} \sigma_{2 q-1}^{A^{\prime}}
$$

where $\varrho_{n}^{11}$ is the restriction of $\varrho_{n}$ on

$$
\left(\frac{\lim }{m} H_{1+2 m}(A)\right) \otimes\left(\underset{\frac{\lim }{m}}{\operatorname{loc}} C_{1+2 m}\left(A^{\prime}\right)\right) .
$$

The result follows since $\operatorname{ker} \varrho_{n}=\operatorname{ker} \varrho_{n}^{00} \oplus \operatorname{ker} \varrho_{n}^{11}$. The case of an odd $n$ is similar.

Proof of assertion (ii). It suffices to show that the inclusion $\operatorname{im} \varrho_{n} \subseteq \operatorname{im} \sigma_{n}^{\square}$ is an equality. This follows as above, using Proposition 2.5(iii).

Proof of assertion (iii). The inclusion $\Delta\left(\operatorname{ker} \sigma_{n}^{A \otimes A^{\prime}}\right) \subseteq \operatorname{ker} \sigma_{n}^{\square}$ follows from the commutativity of (9). In view of assertion (i), in order to show that $\tau\left(\operatorname{ker} \varrho_{n}\right) \subseteq$ $\Delta\left(\operatorname{ker} \sigma_{n}^{A \otimes A^{\prime}}\right)$, we have to show that $\tau\left(\operatorname{ker} \sigma_{p}^{A} \otimes \operatorname{ker} \sigma_{q}^{A^{\prime}}\right) \subseteq \Delta\left(\operatorname{ker} \sigma_{n}^{A \otimes A^{\prime}}\right)$ for all $p, q$ with $p+q=n-2$.

Suppose $n=2 l, p=2 r$ and $q=2 s$ are all even. Let $\Xi \in \operatorname{ker} \sigma_{p}^{A}$ and $\Xi^{\prime} \in \operatorname{ker} \sigma_{q}^{A^{\prime}}$. Then, we can choose cycles $\left(\xi_{m}\right)_{m} \in \prod_{m} \bar{C}_{2 m}(A)$ and $\left(\xi_{m}^{\prime}\right)_{m} \in$ $\prod_{m} \bar{C}_{2 m}\left(A^{\prime}\right)$ (for the differential $b+B$ ) with $\xi_{i}=0$ for $i \leqslant r$ and $\xi_{j}^{\prime}=0$ for $j \leqslant s$, which represent certain elements of $\mathrm{HP}_{0}(A)$ and $\mathrm{HP}_{0}\left(A^{\prime}\right)$ mapping onto $\Xi$ and $\Xi^{\prime}$, respectively. Lemma 3.2(ii) implies that the cycle

$$
\left(\sum_{i+j=m} \xi_{i} \otimes \xi_{j}^{\prime}\right)_{m} \in \prod_{m}\left(\bar{C}(A) \otimes \bar{C}\left(A^{\prime}\right)\right)_{2 m}
$$

represents an element of $\mathrm{HP}_{0}\left(\bar{C}(A) \otimes \bar{C}\left(A^{\prime}\right)\right) \simeq \mathrm{HP}_{0}\left(A \otimes A^{\prime}\right)$ mapping onto $\Delta^{-1} \tau\left(\Xi \otimes \Xi^{\prime}\right)$. Since $r+s=l-1$, we have $\xi_{i} \otimes \xi_{j}^{\prime}=0$ for $i+j \leqslant l$ and, hence, $\sum_{i+j=m} \xi_{i} \otimes \xi_{j}^{\prime}=0$ for $m \leqslant l$. Therefore, $\Delta^{-1} \tau\left(\Xi \otimes \Xi^{\prime}\right) \in \operatorname{ker} \sigma_{n}^{A \otimes A^{\prime}}$.

The cases where $n$ is even and $p, q$ are both odd or $n$ is odd are analogous to the one considered above.

Proof of assertion (iv). We shall need the following lemma. 
LEMMA 3.4. Let $f: M \rightarrow N$ be a map of filtered vector spaces. Suppose that

(i) $M$ is complete and $N$ is Hausdorff with respect to the linear topologies induced by the filtrations,

(ii) $f$ is injective and

(iii) the associated map $\operatorname{gr}(f): \operatorname{gr}(M) \rightarrow \operatorname{gr}(N)$ is surjective.

Then, $\operatorname{gr}(f)$ is injective (and, hence, an isomorphism).

We filter the vector spaces

$$
\frac{\lim }{m} \mathrm{HC}_{*+2 m}\left(A \otimes A^{\prime}\right) \quad \text { and } \underset{m}{\lim }\left(\mathrm{HC}(A) \square \mathrm{HC}\left(A^{\prime}\right)\right)_{*+2 m}
$$

by their subspaces

$$
\operatorname{ker} \sigma_{n}^{A \otimes A^{\prime}},[n]=*, \quad \text { and } \operatorname{ker} \sigma_{n}^{\square},[n]=*,
$$

respectively. As already noted above, assertions (ii) and (iii) imply that the map $\Delta: \mathcal{H}\left(A \otimes A^{\prime}\right) \rightarrow H^{\square}$ is surjective; hence, Lemma 3.4 shows that it is injective as well. This finishes the proof of assertion (iv) and therefore the proof of the theorem.

Remarks 3.5. (i) Let the notations be as in the proof of Theorem 3.3. Then, using the isomorphism $\Delta: \mathcal{H}\left(A \otimes A^{\prime}\right) \rightarrow H^{\square}$, one can show that $\Delta\left(\operatorname{ker} \sigma_{n}^{A \otimes A^{\prime}}\right)=\operatorname{ker} \sigma_{n}^{\square}$ for all $n$.

(ii) One can describe the relation between $\underset{m}{\lim } \mathrm{HC}(A)[-2 m], \underset{m}{\lim } \mathrm{HC}\left(A^{\prime}\right)[-2 m]$ and $\underset{m}{\lim } \mathrm{HC}\left(A \otimes A^{\prime}\right)[-2 m]$ as follows (cf. Remark 2.7(i)).

Let $\mathcal{F}^{A}$ be the filtration of $\underset{m}{\lim } \mathrm{HC}(A)[-2 m]$ given by the kernels of the various maps $\sigma^{A}$ and similarly for $A^{\prime}$ and $A \otimes A^{\prime}$. Then, the tensor product filtration $\mathcal{F}^{A} \dot{\otimes} \mathcal{F}^{A^{\prime}}$ induces a linear topology on

$$
\left(\lim _{m} H C(A)[-2 m]\right) \dot{\otimes}\left(\frac{\lim }{m} H C\left(A^{\prime}\right)[-2 m]\right)
$$

and the corresponding completion is identified with

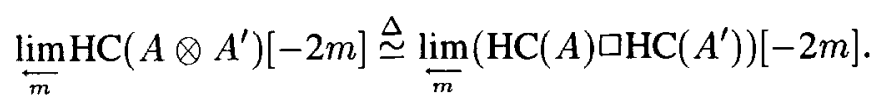

Moreover, under this identification, the completion $\mathcal{F}^{A} \dot{\otimes} \mathcal{F}^{A^{\prime}}$ of $\mathcal{F}^{A} \dot{\otimes} \mathcal{F}^{A^{\prime}}$ corresponds (up to a shift in degrees) to $\mathcal{F}^{A \otimes A^{\prime}}$.

\section{Tensor Products and Periodic Cyclic Homology}

Let $A, A^{\prime}$ be two algebras. In this section, we will show how one can lift the isomorphism of Theorem 3.3 to periodic cyclic homology under the presence 
of certain finiteness hypotheses. More precisely, we shall be interested in the morphism

$\Theta: \mathrm{HP}(A) \dot{\mathrm{HP}}\left(A^{\prime}\right) \rightarrow \mathrm{HP}\left(A \otimes A^{\prime}\right)$

of $\mathbf{Z} / 2 \mathbf{Z}$-graded vector spaces which is induced by (8).

An algebra $A$ will be said to have property (II) if the following conditions are satisfied:

(II1) the inverse system $(\mathrm{HC}(A)[-2 m], S)_{m}$ satisfies the Mittag-Leffler condition and

(П2) the periodic cyclic homology $\operatorname{HP}(A)$ is finite-dimensional.

In particular, if $A$ has property (II), then $\operatorname{HP}(A)=\underset{m}{\lim } \operatorname{HC}(A)[-2 m]$. If $A$ is countably generated as an algebra condition (II2) implies condition (II1); this is a consequence of Gray's criterion for an inverse system to satisfy the Mittag-Leffler condition (cf. [3, Appendix]).

Remarks 4.1. (i) For algebras with property (II), the inverse system $(\mathrm{HC}[-2 m], S)_{m}$ has a particularly simple structure. In fact, one can show that the following conditions are equivalent for an algebra $A$.

$(\alpha) A$ has property (II).

$(\beta)$ The periodic cyclic homology $\operatorname{HP}(A)$ is finite-dimensional and the inverse system $(\mathrm{HC}(A)[-2 m], S)_{m}$ decomposes canonically in sufficiently large degrees into the direct sum of the constant system with value $\operatorname{HP}(A)$ and a system which is essentially zero.

$(\gamma)$ The inverse system $(\mathrm{HC}(A)[-2 m], S)_{m}$ satisfies the Mittag-Leffler condition and the vector space $\mathcal{H}(A)=\oplus_{n} \mathcal{H}_{n}(A)$ is finite-dimensional.

(ii) If an algebra has the property (P) introduced by Kassel in [10], then it also has property $(\Pi)$. If char $k=0$ all commutative algebras of finite type have property ( $\Pi)$, while there are such algebras without property (P) (cf. [3]).

(iii) For algebras with property (II) periodic cyclic homology is compatible with extensions of the base field. In fact, one can show that the following conditions are equivalent for an algebra $A$ (cf. [4, Remark 8(ii)]).

$(\alpha)$ The canonical map $\operatorname{HP}(A) \otimes K \rightarrow \operatorname{HP}\left(A^{K}\right)$ is an isomorphism for any field extension $K / k$, where we denote by $A^{K}$ the $K$-algebra $A \otimes K$.

$(\beta)$ The inverse system $(\mathrm{HC}(A)[-2 m], S)_{m}$ satisfies the Mittag-Leffler condition and $\sigma_{n}^{A}\left(=\bar{\sigma}_{n}^{A}\right)$ is injective for $n \gg 0$.

$\left(\beta^{\prime}\right)$ The inverse system $(\mathrm{HC}(A)[-2 m], S)_{m}$ satisfies the Mittag-Leffler condition and $\mathcal{H}_{n}(A)=0$ for $n \gg 0$.

In particular, if $A$ has property (II), then so does the $K$-algebra $A^{K}=A \otimes K$ for any field extension $K / k$.

The next result is a generalization of [10, Theorem 3.10]. 
THEOREM 4.2. The following two conditions are equivalent for an algebra $A$.

(i) A has property (II).

(ii) $\Theta: \mathrm{HP}(A) \otimes \mathrm{HP}\left(A^{\prime}\right) \rightarrow \mathrm{HP}\left(A \otimes A^{\prime}\right)$ is an isomorphism for any algebra $A^{\prime}$.

Proof. (i) $\rightarrow$ (ii) Let $A^{\prime}$ be an algebra. For all $m$ we shall use the following notations:

$$
\begin{aligned}
& X_{m}=\mathcal{B}(\bar{C}(A))[-2 m], \quad X_{m}^{\prime}=\mathcal{B}\left(\bar{C}\left(A^{\prime}\right)\right)[-2 m], \\
& Y_{m}=\mathcal{B}\left(\bar{C}(A) \otimes \bar{C}\left(A^{\prime}\right)\right)[-2 m], \quad Z_{m}=\left(\mathcal{B}(\bar{C}(A)) \otimes \mathcal{B}\left(\bar{C}\left(A^{\prime}\right)\right)\right)[-2 m] .
\end{aligned}
$$

We view $\left(Z_{m}\right)_{m}$ as a surjective inverse system of complexes with respect to $S \otimes 1$ and consider the commutative diagram of supercomplexes with exact rows

$0 \longrightarrow\left(\underset{\frac{\lim }{m}}{X_{m}}\right) \dot{\otimes}\left(\underset{\frac{\lim }{m}}{X_{m}^{\prime}}\right) \longrightarrow\left(\underset{\frac{\lim }{m}}{ } X_{m}\right) \dot{\otimes}\left(\prod_{m} X_{m}^{\prime}\right) \longrightarrow\left(\underset{\frac{\lim }{m}}{ } X_{m}\right) \dot{\otimes}\left(\prod_{m} X_{m}^{\prime}\right) \longrightarrow 0$

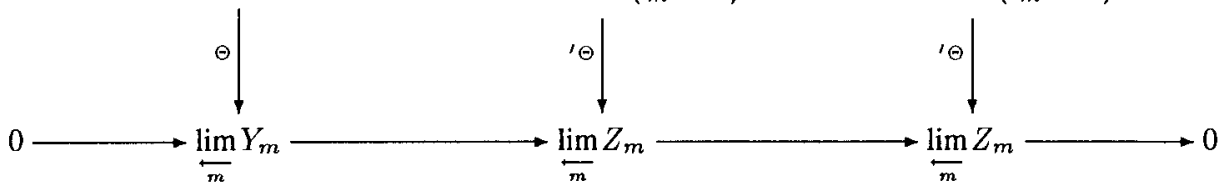

which is defined as follows. The top row is obtained by tensoring with $\underset{m}{\lim } X_{m}$ the short exact sequence

$$
0 \rightarrow \underset{m}{\lim } X_{m}^{\prime} \rightarrow \prod_{m} X_{m}^{\prime} \stackrel{1-S}{\longrightarrow} \prod_{m} X_{m}^{\prime} \rightarrow 0
$$

the bottom row is obtained from (6) by passing to inverse limits and ' $\Theta$ is the restriction of the morphism $\left(\prod_{m} X_{m}\right) \dot{\otimes}\left(\prod_{m} X_{m}^{\prime}\right) \rightarrow\left(\prod_{m} Z_{m}\right)$ which maps $\left(x_{m}\right)_{m} \otimes\left(x_{m}^{\prime}\right)_{m}$ onto $\left(\sum_{i+j=m} x_{i} \otimes x_{j}^{\prime}\right)_{m}$.

The homology of $Z_{m}$ is $\left(\mathrm{HC}(A) \otimes \mathrm{HC}\left(A^{\prime}\right)\right)[-2 m]$ and, hence, assumption (I1) implies that the inverse system $\left(H_{*}\left(Z_{m}\right), S \otimes 1\right)_{m}$ satisfies the Mittag-Leffler condition; it follows that

$$
H_{*}\left(\frac{\lim }{m} Z_{m}\right)=\frac{\lim }{m} H_{*}\left(Z_{m}\right)=\frac{\lim }{m}\left(\mathrm{HC}(A) \otimes \mathrm{HC}\left(A^{\prime}\right)\right)_{*+2 m} .
$$

We note that ${ }^{\prime} \Theta$ induces an isomorphism

$$
\begin{aligned}
& \left(\mathrm{HP}(A) \dot{\otimes} \prod_{m} \mathrm{HC}\left(A^{\prime}\right)[-2 m]\right)_{*} \\
& \simeq \prod_{l}\left(\mathrm{HP}_{*-[l]}(A) \otimes \mathrm{HC}_{l}\left(A^{\prime}\right)\right)
\end{aligned}
$$




$$
\begin{aligned}
& \simeq \prod_{l}\left(\left(\frac{\lim H_{m}}{*-l+2 m}(A)\right) \otimes \mathrm{HC}_{l}\left(A^{\prime}\right)\right) \\
& \simeq \prod_{l}{\underset{m}{\lim }\left(\mathrm{HC}_{*-l+2 m}(A) \otimes \mathrm{HC}_{l}\left(A^{\prime}\right)\right)}=\lim _{m}\left(\mathrm{HC}(A) \otimes \mathrm{HC}\left(A^{\prime}\right)\right)_{*+2 m}
\end{aligned}
$$

where we have used (П2), (П1) and [4, Proposition 2]. Using the 5-lemma, we conclude that $\Theta$ induces an isomorphism in homology as well.

(ii) $\rightarrow$ (i) In order to show that $A$ satisfies condition (II), we consider an algebra $A^{\prime}$ which is not finite-dimensional as a vector space such that the projection $\left(\bar{C}\left(A^{\prime}\right), b\right) \rightarrow A^{\prime}[0]$ is a quasi-isomorphism (e.g., $A^{\prime}$ can be the ind-étale algebra which consists of all eventually constant sequences in $k$ with addition and multiplication defined component-wise). Then, there are induced quasi-isomorphisms of supercomplexes

$$
\underset{m}{\lim } \mathcal{B}\left(\bar{C}\left(A^{\prime}\right)\right)[-2 m] \rightarrow A^{\prime}[0]
$$

and

$$
\underset{m}{\lim } \mathcal{B}\left(\bar{C}(A) \otimes \bar{C}\left(A^{\prime}\right)\right)[-2 m] \rightarrow \underset{m}{\rightarrow} \underset{\lim }{m}\left(\mathcal{B}(\bar{C}(A))[-2 m] \otimes A^{\prime}[0]\right)
$$

such that $\Theta$ descends to the canonical map

$$
\Phi:\left(\lim _{m} \mathcal{B}(\bar{C}(A))[-2 m]\right) \dot{\otimes} A^{\prime}[0] \rightarrow \underset{m}{\lim }\left(\mathcal{B}(\bar{C}(A))[-2 m] \otimes A^{\prime}[0]\right) .
$$

Since $\Phi$ is a quasi-isomorphism, one can use the results of [4] and show that the inverse system $(\mathrm{HC}(A)[-2 m], S)_{m}$ satisfies the Mittag-Leffler condition (cf. [4, Remark 8(ii)]).

In order to show that $A$ satisfies condition (II2), we consider an algebra $A^{\prime}$ such that $\operatorname{ker} \sigma_{n}^{A^{\prime}} \neq 0$ for all $n$. (An example of such an algebra can be obtained as follows. We start with a (commutative) smooth $k$-algebra $R$ with $H_{d R}^{1}(\operatorname{Spec} R) \neq 0$ and consider for all $t \in \mathbf{N}$ the inclusions $R^{\otimes t}=R^{\otimes t} \otimes k \hookrightarrow R^{\otimes t} \otimes R=R^{\otimes t+1}$. One can show that $A^{\prime}=\underset{\vec{t}}{\lim } R^{\otimes t}$ has the required property.) Since the isomorphism $\Theta$ descends to $\Delta^{-1} \tau$ (Lemma 3.2(ii)), we conlude that $\tau$ is surjective and, hence, an isomorphism. It remains to invoke Remark 2.7(ii).

Remark 4.3. In the proof of the implication (ii) $\rightarrow$ (i) of Theorem 4.2, we have exhibited examples of algebras which do not have property ( $\Pi$ ). In fact, the product morphism $\Theta$ is not an isomorphism when $A$ is the algebra of all eventually constant sequences in $k$ and $A^{\prime}$ the direct limit of the tensor powers of a smooth algebra whose first de Rham cohomology group is nontrivial. 
Since commutative algebras of finite type in characteristic 0 have property (II), the computation of their periodic cyclic homology by Feigin and Tsygan (cf. [5]) yields the following

COROLLARY 4.4. Suppose that char $k=0$ and let $A$ be a commutative algebra of finite type. Then, for any algebra $A^{\prime}$, there is a canonical isomorphism

$$
\mathrm{HP}\left(A \otimes A^{\prime}\right) \simeq H_{\mathrm{inf}}(\operatorname{Spec} A) \dot{\otimes} \operatorname{HP}\left(A^{\prime}\right)
$$

of $\mathbf{Z} / 2 \mathbf{Z}$-graded vector spaces, where the infinitesimal cohomology $H_{\mathrm{inf}}(\operatorname{Spec} A)$ of Spec $A$ over Spec $k$ is viewed with the even-odd grading.

Finally, using Theorem 4.2, we show that the class of algebras having property (II) is closed under tensor products.

PROPOSITION 4.5. Let $A, A^{\prime}$ be two algebras.

(i) If $A$ and $A^{\prime}$ have property (II1) then so does $A \otimes A^{\prime}$.

(ii) If $A$ and $A^{\prime}$ have property (II) then so does $A \otimes A^{\prime}$.

Proof. (i) Let us consider the short exact sequence (7). Since the Mittag-Leffler condition is stable under extensions of inverse systems (as one can verify by a diagram chase), the result follows from Lemma 2.1(i) and Proposition 2.3.

(ii) This follows from (i) and Theorem 4.2.

\section{References}

1. Burghelea, D. and Ogle, C.: The Künneth formula in cyclic homology, Math. Z. 193 (1986), 527-536.

2. Connes, A.: Noncommutative differential geometry, Publ. Math. IHES 62 (1985), 41-144.

3. Emmanouil, I.: The cyclic homology of affine algebras, Ph.D, thesis, University of California, Berkeley, 1994.

4. Emmanouil, I.: Mittag-Leffler condition and the vanishing of $\lim ^{1}$ (To appear in Topology.)

5. Feigin, B. L. and Tsygan, B. L.: Additive $K$-theory and crystalline cohomology, Funct. Anal. Appl. 19 (1985), 124-132.

6. Goodwillie, T.: Cyclic homology, derivations and the free loopspace, Topology 24 (1985), 187-215.

7. Grothendieck, A.: Crystals and the de Rham cohomology of schemes, notes by J. Coates and D. Jussila, Dix exposés sur la cohomologie des schémas, North-Holland, Amsterdam, 1968.

8. Hilton, P. J. and Stammbach, U.: A Course in Homological Algebra, Grad. Texts in Math. 4, Springer, Berlin, Heidelberg, New York, 1971.

9. Karoubi, M.: Formule de Künneth en homologie cyclique I, II, C.R. Acad. Sci. Paris 303 (1986), 527-530, 595-598.

10. Kassel, C.: Cyclic homology, comodules and mixed complexes, J. Algebra 107 (1987), 195-216.

11. Loday, J.-L.: Cyclic Homology, Grundl. Math. Wiss. 301, Springer, Berlin, Heidelberg, New York, 1992.

12. Loday, J.-L. and Quillen, D.: Cyclic homology and the Lie algebra homology of matrices, Comment. Math. Helv. 59 (1984), 565-591.

13. Vigué-Poirrier, M.: Cyclic homology of algebraic hypersurfaces, J. Pure Appl. Algebra 72 (1991), 95-108. 